\title{
Levamisole in Children with Idiopathic Nephrotic Syndrome: Clinical Efficacy and Pathophysiological Aspects
}

\author{
Anne K. Mühlig ${ }^{1, \dagger}$, Jun Young Lee ${ }^{2,+}{ }^{\oplus}$, Markus J. Kemper ${ }^{3}$, Andreas Kronbichler ${ }^{4}{ }^{\oplus}$, \\ Jae Won Yang ${ }^{2}{ }^{(0}$, Jiwon M. Lee ${ }^{5}\left(\mathbb{D}\right.$, Jae Il Shin ${ }^{6,7,8, *(\mathbb{C})}$ and Jun $\mathrm{Oh}^{1}{ }^{1}$ \\ 1 Department of Pediatrics, University Hamburg-Eppendorf, 20246 Hamburg, Germany; \\ a.dettmar@uke.de (A.K.M.); j.oh@uke.de (J.O.) \\ 2 Department of Nephrology, Yonsei University Wonju College of Medicine, Wonju, Kangwon 26426, Korea; \\ junyoung07@yonsei.ac.kr (J.Y.L.); kidney74@yonsei.ac.kr (J.W.Y.) \\ 3 Department of Pediatrics, Asklepios Klink Nord-Heidberg, 22417 Hamburg, Germany; \\ m.kemper@asklepios.com \\ 4 Department of Internal Medicine IV (Nephrology and Hypertension), Medical University Innsbruck, \\ Innsbruck 6020, Austria; andreas.kronbichler@i-med.ac.at \\ 5 Department of Pediatric Nephrology, Chungnam National University Hospital, Daejeon 35015, Korea; \\ jwmleemd@gmail.com \\ 6 Department of Pediatrics, Yonsei University College of Medicine, Seoul 03722, Korea \\ 7 Division of Pediatric Nephrology, Severance Children's Hospital, Seoul 03722, Korea \\ 8 Institute of Kidney Disease Research, Yonsei University College of Medicine, Seoul 03722, Korea \\ * Correspondence: shinji@yuhs.ac; Tel.: +82-2-228-2050; Fax: +82-2-393-9118 \\ + These authors contributed equally to this work.
}

Received: 30 May 2019; Accepted: 12 June 2019; Published: 16 June 2019

Abstract: Steroid sensitive nephrotic syndrome is one of the most common pediatric glomerular diseases. Unfortunately, it follows a relapsing and remitting course in the majority of cases, with 50\% of all cases relapsing once or even more often. Most children with idiopathic nephrotic syndrome respond initially to steroid therapy, nevertheless repeated courses for patients with relapses induce significant steroid toxicity. Patients with frequent relapses or steroid dependency thus require alternative treatment, such as cyclophosphamide, cyclosporine, tacrolimus, mycophenolate mofetil, levamisole, or rituximab. To reduce the relapse rate, several drugs have been used. Among these, levamisole has been considered the least toxic and least expensive therapy. Several randomized controlled trials (RCT) showed that levamisole is effective in reducing the relapse risk in steroid sensitive forms of nephrotic syndrome with a low frequency of side effects. Levamisole is a synthetic imidazothiazole derivative with immune-modulatory properties. In this article, we review recent data from randomized trials and observational studies to assess the efficacy of levamisole in frequently relapsing nephrotic syndrome and steroid-dependent nephrotic syndrome.

Keywords: levamisole; nephrotic syndrome; podocyte; steroid-dependent nephrotic syndrome

\section{Introduction}

Nephrotic syndrome (NS) occurs in 16 out of 100,000 children in western countries and is one of the most common renal diseases in the pediatric population [1]. It is characterized by the appearance of proteinuria $\left(>1 \mathrm{~g} / \mathrm{m}^{2} /\right.$ day), hypoalbuminemia $(<25 \mathrm{~g} / \mathrm{L})$, generalized edema and normal glomerular function. Usually, there is an idiopathic NS (iNS) with two histological subtypes: (1) NS with small histological changes, so-called minimal change nephrotic syndrome (MCNS); and with more pronounce histological lesions, (2) focal segmental glomerulosclerosis (FSGS). 
Idiopathic nephrotic syndrome (iNS) seems to be a T-cell-mediated disease. Clinical observations show that the first episode and relapses often develop after an infection of the upper respiratory tract or an allergic reaction. In the acute phase of the disease, a shift in lymphocyte population has been reported [2]. There also seems to be an enhanced type II immunologic reaction in inactive forms of NS [3]. These findings and others indicate that there has to be an immunologic trigger in patients with iNS.

Centrally involved in the onset of proteinuria are podocytes [4]. Podocytes are highly specialized, terminally differentiated cells, forming the glomerular filtration barrier together with the glomerular basement membrane and glomerular endothelial cells. Effacement of podocyte foot processes can be seen in electron microscopy, which is the only abnormality seen in MCNS.

Treatment with steroid is still golden standard, and most forms (90\%) of NS show remission within four weeks of treatment with steroids. In cases who respond to steroids, iNS is classified as steroid-sensitive nephrotic syndrome (SSNS), defined by the International Study of Kidney Disease in Children (ISKDC) [5].

The overall prognosis of children suffering from NS is good. Usually, the disease generally disappears at the onset of puberty. Nevertheless, up to $85 \%$ develop at least one relapse within the next five years [6]. Relapse risk generally decreases over time, but is prolonged during adulthood in some cases [7]. Recent research focuses on factors leading to initiation of iNS, the prediction of recurrence rate and important factors to reduce the risk of relapse. However, the exact mechanism of iNS are still elusive.

\section{Side Effects of Traditional Immunosuppressive Agents in the Treatment of Nephrotic Syndrome}

Regarding the literature, approximately $30-60 \%$ of all children suffering from a SSNS are frequently relapsing or even steroid-dependent forms of NS [8,9]. Frequently relapsing nephrotic syndrome (FRNS) is defined as more than four relapses in one year or more than two relapses following the first six months after the initial presentation. Patients with at least two relapses during treatment with alternate-day steroids or within 14 days after stopping steroid treatment are classified as steroid-dependent (SDNS) [8,10]. Treatment of relapses is usually based on steroids with a shorter duration (prednisone $60 \mathrm{mg} / \mathrm{m}^{2}$ until remission, 4 weeks $40 \mathrm{mg} / \mathrm{m}^{2}$ every other day) [5]. FRNS and SDNS usually react quickly to treatment with glucocorticoids, and long-term prognosis regarding renal function is very good [11], but patients suffer from relapses frequently and need to be treated repeatedly. However, cushingoid changes, hyperglycemia, infection, abnormal bone metabolism, skin atrophy, striae, peptic ulcer, adrenal suppression, increased blood pressure, and behavioral changes are well-recognized, potentially serious adverse effects of corticosteroids [12-14].

Therefore, there is an increasing demand for alternative less harmful therapies. In the past, there has been extensive evidence for the use of so-called steroid-sparing agents such as mycophenolic acid (MPA), rituximab, alkylating agents like cyclophosphamide and calcineurin inhibitors, which have been summarized and reviewed elsewhere $[5,15]$. Cyclophosphamide has bladder toxicity and other side effects such as gonadal toxicity, bone marrow depression, carcinogenesis, and an increased risk of infection [16]. Calcineurin inhibitors, cyclosporine and tacrolimus may lead to neurotoxicity, hirsutism, gingival hyperplasia, and nephrotoxicity [17]. Mycophenolate mofetil (MMF) and MPA are newer immunosuppressive drugs that have fewer serious adverse effect but have the disadvantage of high therapy costs $[18,19]$. 
The aim of this review is to focus on the use of levamisole, which seems to have a similar effect with other substances, but exhibits a lower number of adverse events and reactions. Levamisole has been considered the least toxic and least expensive steroid-sparing drug for preventing relapses of SSNS. Therefore, the use of levamisole should always be taken into account. However, levamisole is used in restricted areas (mostly in India and Europe). In FRNS and SDNS, levamisole significantly reduces both the relapse rate and the cumulative steroid dose; therefore, it should be recommended for these patients. In this review, we focus on the efficacy by critically discussing the literature, including randomized controlled trials (RCT), observational studies and existing meta-analyses for levamisole in order to highlight the usefulness and side effects of the drug, and the implications to treatment for nephrotic syndrome.

\section{The Use of Levamisole in the Treatment of Nephrotic Syndrome}

Levamisole is an immune-modulating imidazothiol-derived anthelminthic. It was first used in 1969 as an anthelmintic agent. In humans, levamisole was originally used in the treatment of leprosy [20] and colonic carcinoma [21]. As a treatment of SSNS, it has been used since the early 1980s [22]. Besides a few uncontrolled levamisole studies [23,24], the first controlled study was performed by the British Association of Pediatric Nephrology, published in 1991. In this study, a significant reduction of disease relapses in children was demonstrated with the use of levamisole [25]. Data from Kemper et al. identified that patients with exclusively frequent relapses responded to levamisole better than those with steroid dependence [26]. The Indian pediatric nephrology group revised its guidelines of nephrotic syndrome so that levamisole could be used as a steroid-sparing agent. The recommended dose is $2-2.5 \mathrm{mg} / \mathrm{kg}$ on alternate days for $12-24$ months, while monitoring leukocyte count every 12-16 weeks [27]. In 2017, Gruppen et al. published an international RCT. In this double-blinded study, 99 children with SDNS and FRNS were treated with levamisole $(2.5 \mathrm{mg} / \mathrm{kg}$ on alternate days) or placebo. They found that there was a significantly longer time to the first relapse in patients treated with levamisole compared to the placebo group [28]. Subgroup analysis showed that cases with SDNS (mostly from Europe) had an inferior response to FRNS (mostly from India). There are some considerations preferring levamisole in the treatment of FRNS over its use in SDNS [20,29]. The authors discussed different genetic backgrounds and immunological changes due to steroid dependency and preceding long-term steroid treatments, resulting in pharmacological interactions [28,29]. Recently in an open-label RCT studying SDNS and FRNS, both MMF (76 patients with $750-1000 \mathrm{mg} / \mathrm{m}^{2}$ ) and levamisole (73 patients with 2 to $2.5 \mathrm{mg} / \mathrm{kg}$ on alternate days) showed similar relapse rates, treatment failure rates, disease course and decreased cumulative steroid dose [30]. As $2.5 \mathrm{mg} / \mathrm{kg}$ every other day is the historical dosage, more studies are needed to define the appropriate dosage and duration of treatment [29].

There are several older RCTs, which were summarized in a Cochrane analysis published in 2013 [31]. When treatment with levamisole was compared to placebo, low-dose prednisone or no treatment, there was a significant reduction of relapse numbers in children with SSNS. Several different levamisole dosages have been employed in terms of total dose administrated ( $35 \mathrm{mg} / \mathrm{m}^{2} / \mathrm{month}$ vs. $20 \mathrm{mg} / \mathrm{m}^{2} / \mathrm{month}$ ) or frequency (alternate day vs. 2 consecutive days of 7 days) [32]. The risk of relapse during the treatment period decreased by up to $10-45 \%$ in various studies [33]. However, the duration of treatment was relatively short; it varied from four to twelve months. Another observation was that levamisole reduced the relapse rate only during the actual treatment period, and had no significant impact on relapse risk after the discontinuation. Compared to intravenous, monthly [34] or orally administered cyclophosphamide [31], there seems to be a slightly higher rate of relapse during levamisole therapy. Nevertheless, there was no significant difference in the number of children suffering from one or more relapses in the period between both treatments [24]. 
In addition to these RCTs, there have been a lot of smaller retrospective studies in the last decade, but most often only single center experiences have been published [35-40]. These papers stated that in children with FRNS or SDNS [36-38,40], levamisole could be effective in decreasing relapse risk and frequency. However, some of these studies used low-dose prednisolone or a slowly tapering regimen in addition to $2-2.5 \mathrm{mg} / \mathrm{kg}$ body weight levamisole on alternate days [27]. Many of these studies showed that levamisole lowered the number of relapses during the therapy period. Additionally, the use of steroids and the total cumulative dosage were significantly reduced. Interestingly, in one study, the relapse rate was even higher after the discontinuation of the treatment [39], whereas Elmas et al. reported a reduced relapse risk 12 month after cessation of levamisole [40]. Basu and colleagues [39] retrospectively analyzed the data from children who had received either levamisole or MMF or tacrolimus. They verified that the relapse-free survival was higher after 30 months in patients treated with tacrolimus or MMF compared to levamisole ( $61.7 \%$ vs. $38.5 \%$ vs. $24 \%)$. Nevertheless, levamisole treatment resulted in a significant reduction in relapse rate compared to the relapse frequency in the year before treatment.

Tables 1 and 2 summarized recent reports of levamisole for FRNS or SDNS in children and adults. As stated above, levamisole has been commonly used at $2.5 \mathrm{mg} / \mathrm{kg} /$ day every other day (maximum $150 \mathrm{mg})$. In all studies, levamisole was given at a dose of $35 \mathrm{mg} / \mathrm{kg} / \mathrm{month}(2-2.5 \mathrm{mg} / \mathrm{kg} /$ day every other day), while a lower dosage seems to be less effective [31]. Some retrospective studies used levamisole every day. Many observational studies have been conducted, mainly to show the reduction of the recurrence rate in the treatment period after levamisole initiation compared with the time before levamisole. Although not many RCTs and meta-analyses had yet been performed, these studies also showed that levamisole reduced recurrence rate compared with placebo or steroid monotherapy. Some RCTs showed that levamisole was not inferior to MMF and cyclophosphamide. A meta-analysis was not sufficient to compare levamisole to MMF and cyclophosphamide (Table 3). We were not able to provide any data on accurate dose, timing, and duration of levamisole. The pharmacokinetic profile in children seems to be similar to the data available for adults, except for a documented slightly higher clearance rate in children [41]. A French trial (NEPHROVIR 3, NCT02818738) comparing the effects of levamisole with steroid at initial INS presentation for reducing relapse rate is currently ongoing. 
Table 1. Observational studies of steroid-dependent or frequent relapsing nephrotic syndrome treated with levamisole.

\begin{tabular}{|c|c|c|c|c|c|c|}
\hline Author, Year & $\begin{array}{c}\text { Study } \\
\text { Design }\end{array}$ & $\begin{array}{c}\text { Comparison (Dose of } \\
\text { Levamisole) }\end{array}$ & $\begin{array}{c}\text { Total } \\
\text { Patients }\end{array}$ & Relapse (\%) & FU & Other Effect \\
\hline Tanphaichitr P, 1980 [22] & Obs & (1.5-3.9 mg/kg twice per week) & 7 & - & $1-6 \mathrm{mo}$ & $(33.5 \pm 9.5 \% \text { vs. } 69.3 \pm 3.9 \%)^{a}$ \\
\hline Niaudet P, 1984 [42] & Obs & (2.5 mg/kg twice per week) & 30 & - & $9.9 \mathrm{mo}$ & - \\
\hline Mehta KP, 1986 [43] & Obs & $(2.5 \mathrm{mg} / \mathrm{kg}$ qod or $\mathrm{qd})$ & 14 & - & 6-24 mo & 6 Pts CR, 6 Pts PR \\
\hline La Manna A, 1988 [44] & Obs & $(2.5 \mathrm{mg} / \mathrm{kg}$ twice a week or $\mathrm{qd})$ & 13 & - & $7-29 \mathrm{mo}$ & $\mathrm{b}$ \\
\hline Srivastava RN, 1991 [45] & Obs & $(5 \mathrm{mg} / \mathrm{kg}$ qod $)$ & 12 & Before L (3.3/yr), After L (2.3/yr) & $3 \mathrm{mo}$ & - \\
\hline Meregalli P, 1994 [46] & Obs & (5 mg/kg weekly) & 10 & Reduction SD 62-75\% & - & - \\
\hline Ksiazek J, 1995 [47] & Obs & - & 22 & $18.2 \%$ relapse $/ 36.4 \%$ not response & - & $45.5 \%$ remission maintained \\
\hline Ginevri F, 1996 [48] & Obs & (2.5 mg/kg qod) & 20 & $\begin{array}{l}45.5 \% \text { remission maintained after } \mathrm{L} \text {, } \\
36.4 \% \text { not respond to } \mathrm{L}\end{array}$ & - & - \\
\hline Bagga A, 1997 [23] & Obs & (2.5 mg/kg qod) & 43 & Before L (5.2/yr), After 2 years L (0.69/yr) & $6-31 \mathrm{mo}$ & - \\
\hline Kemper MJ, 1998 [26] & Obs & $(2.0 \mathrm{mg} / \mathrm{kg}$ qod $)$ & 25 & $\begin{array}{l}\text { Prior to L } 0.5 / \text { month, } \\
\text { During L } 0.3 / \text { month }\end{array}$ & 3-24 mo & - \\
\hline Fu LS, 2000 [49] & Obs & (2-3 mg/kg qod) & 27 & $\begin{array}{l}\text { Prior to L }(5.74 \pm 3.24 / \mathrm{yr}) \\
\text { During L }(1.91 \pm 2.0 / \mathrm{yr})\end{array}$ & $6-24$ mo & - \\
\hline Alsaran K, 2001 [50] & Obs & $\mathrm{L}(2.5 \mathrm{mg} / \mathrm{kg})$ vs. CPA & 24 & $\begin{array}{l}\text { L ( }(0.28 / \text { month }) \\
\text { CPA ( } 0.32 / \text { month })\end{array}$ & - & c \\
\hline Donia AF, 2002 [51] & Obs & (2.5 mg/kg qod) & 20 & $\begin{array}{l}25 \% \text { in remission } 12 \mathrm{mo} \text {, } \\
75 \% \text { relapses during } \mathrm{Tx}\end{array}$ & $12 \mathrm{mo}$ & - \\
\hline Al-Ibrahim AA, 2003 [52] & Obs & (2.5 mg/kg qod) & 24 & $\begin{array}{l}\text { Prior to L }(4 / y r), \\
\text { During L }(1.3 / y r)\end{array}$ & $12-24 \mathrm{mo}$ & - \\
\hline Sumegi V, 2004 [53] & Obs & $(2 \mathrm{mg} / \mathrm{kg} \mathrm{qd})$ & 34 & $\begin{array}{l}\text { Prior to L }(4.41 / \mathrm{yr}) \text {, } \\
\text { During L }(0.41 / \mathrm{yr})\end{array}$ & $24 \mathrm{mo}$ & $\begin{array}{l}\text { Cumulative steroid dose } \\
7564 \mathrm{mg} / \mathrm{yr} \text { vs. } 1472 \mathrm{mg} / \mathrm{yr}\end{array}$ \\
\hline Fu LS, 2004 [54] & Obs & $(2-3 \mathrm{mg} / \mathrm{kg}$ qod or $\mathrm{qd})$ & 36 & $\begin{array}{l}\text { qod } 2.01 \pm 2.5 / \mathrm{yr}, \\
\text { qd } 1.34 \pm 2.1 / \mathrm{yr}\end{array}$ & $4-36$ mo & - \\
\hline Hafeez F, 2006 [55] & Obs & (2.5 mg/kg qod) & 70 & $19(27.14 \%)$ pts did not relapse on $\mathrm{Tx}$ & $12 \mathrm{mo}$ & $\mathrm{d}$ \\
\hline Boyer O, 2008 [37] & Obs & ( $2.5 \mathrm{mg} / \mathrm{kg} 3$ days/week) & 10 & $\begin{array}{l}\text { Prior to L }(6.0 / y r), \\
\text { During L }(0.0 / y r)\end{array}$ & $48 \mathrm{mo}$ & e \\
\hline Madani A, 2010 [38] & Obs & (2.5 mg/kg qod) & 304 & $\begin{array}{l}\text { Prior to L }(2.0 / y r) \text {, } \\
\text { During L }(1.1 / y r)\end{array}$ & $1-22$ yrs & $\begin{array}{c}\text { Steroid dose was significantly } \\
\text { reduced }\end{array}$ \\
\hline Chen SY, 2010 [56] & Obs & $(2-3.3 \mathrm{mg} / \mathrm{kg} \mathrm{qd})$ & 15 & 1 pt CR, 10 pts No effect & 3-20 mo & - \\
\hline
\end{tabular}


Table 1. Cont.

\begin{tabular}{|c|c|c|c|c|c|c|}
\hline Author, Year & $\begin{array}{l}\text { Study } \\
\text { Design }\end{array}$ & $\begin{array}{c}\text { Comparison (Dose of } \\
\text { Levamisole) }\end{array}$ & $\begin{array}{c}\text { Total } \\
\text { Patients }\end{array}$ & Relapse (\%) & FU & Other Effect \\
\hline Elmas AT, 2013 [40] & Obs & ( $2.5 \mathrm{mg} / \mathrm{kg} 3$ days/week) & 29 & $\begin{array}{l}\text { Prior to L }(4.0 / \mathrm{yr}) \text {, } \\
\text { During L }(0.0 / \mathrm{yr})\end{array}$ & $12 \mathrm{mo}$ & Proteinuria, annual SD reduced \\
\hline Ekambaram S, 2014 [35] & Obs & $(2 \mathrm{mg} / \mathrm{kg} \mathrm{qd})$ & 97 & $\begin{array}{l}\text { Prior to L (2.4/yr), } \\
\text { During L (1.3/yr) }\end{array}$ & $6-24 \mathrm{mo}$ & $\begin{array}{l}\text { Steroid dose }\left(4.1 \mathrm{~g} / \mathrm{m}^{2} \text { to } 2.5\right. \\
\left.\mathrm{g} / \mathrm{m}^{2}\right)\end{array}$ \\
\hline Skrzypczyk P, 2014 [57] & Obs & $\begin{array}{c}(2.5 \mathrm{mg} / \mathrm{kg} / \mathrm{qd} \text { or qod or twice a } \\
\text { week) }\end{array}$ & 21 & - & - & f \\
\hline Jiang L, 2015 [58] & Obs & $(1.25 \mathrm{mg} / \mathrm{kg} \mathrm{qd})$ & 15 & $\begin{array}{l}\text { Adult } 14 \text { pts reduction or stopping other } \\
\text { medications }\end{array}$ & $1-14 \mathrm{yrs}$ & \\
\hline $\begin{array}{l}\text { Kuzma-Mroczkowska E, } \\
2016[36]\end{array}$ & Obs & $(2.5 \mathrm{mg} / \mathrm{kg}$ qod $)$ & 53 & $\begin{array}{l}\text { Prior to L }(2.7 / \mathrm{yr}) \text {, } \\
\text { During L (1.8/yr) }\end{array}$ & $36 \mathrm{mo}$ & $\begin{array}{c}8.8 \text { mo to relapse on levamisole } \\
\text { Tx }\end{array}$ \\
\hline Alsaran, K, 2017 [59] & Obs & $(2.5 \mathrm{mg} / \mathrm{kg}$ qod $)$ & 20 & $\begin{array}{l}\text { Prior to L (3.6/yr), } \\
\text { During L (1.6/yr) }\end{array}$ & $12 \mathrm{mo}$ & $\begin{array}{l}\text { Cumulative steroid dose was } \\
\text { reduced }\end{array}$ \\
\hline $\begin{array}{c}\text { Abeyagunawardena, } 2017 \\
\text { [60] }\end{array}$ & Obs & $(2.5 \mathrm{mg} / \mathrm{kg}$ qod vs. qd) & 58 & $\begin{array}{l}\text { L qod (163/yr), } \\
\text { L qd (77/yr) }\end{array}$ & $12 \mathrm{mo}$ & $\begin{array}{l}\text { M annual SD ( } \mathrm{mg} / \mathrm{kg} / \mathrm{yr}) \text { qod } \\
\text { vs. qd (254 vs. 154) }\end{array}$ \\
\hline Basu B, 2017 [39] & Obs & (2.5 mg/kg qod) & 129 & $\begin{array}{c}\text { During L (1.7/yr) } \\
\text { After L (2.8/yr) }\end{array}$ & $30 \mathrm{mo}$ & $\mathrm{g}$ \\
\hline
\end{tabular}

CPA: Cyclophosphamide, CR: complete response, FU: Follow up, L: Levamisole, M: mean, mo: months, Obs: observational study, PR: partial response, Pt: patient, qd: once daily, qod: every other day, SD: steroid dose, Tx: treatment, yr: year. ${ }^{\text {a } \%}$ of E-rosette before and after levamisole; ${ }^{b}$ Response rate: levamisole $2.5 \mathrm{mg}$ twice a week-4 of the 13 patients, levamisole $2.5 \mathrm{mg}$ qd-2 patients of the 8 patients; ${ }^{\mathrm{c}}$ Levamisole $(0.28)$ and cyclophosphamide $(0.32)$ showed no significant difference in reduction of relapse rate; ${ }^{\mathrm{d}}$ Levamisole was more effective in older children ( $>5$ years, vs. $<5$ years); ${ }^{\text {e }}$ cumulative steroid $6067 \mathrm{mg} / \mathrm{m}^{2}$ to $2920 \mathrm{mg} / \mathrm{m}^{2}$; ${ }^{\mathrm{f}}$ Responder group was more frequently treated with steroid and levamisole and less frequently treated with cyclosporine $\mathrm{A} ;{ }^{\mathrm{g}}$ The relapse free survival was higher with tacrolimus than levamisole $(61.7 \%$ vs. $24 \%)$. 
Table 2. Randomized controlled trials of steroid-dependent or frequent relapsing nephrotic syndrome children treated with levamisole (RCT).

\begin{tabular}{|c|c|c|c|c|c|c|c|}
\hline Author, Year & Inclusion & Exclusion & Comparison & $\begin{array}{c}\text { Total } \\
\text { Patients/Control } \\
\end{array}$ & Relapse (\%) & $\begin{array}{c}\mathrm{FU} \\
\text { Weeks }\end{array}$ & $\begin{array}{l}\text { Effect Size } \\
(\mathrm{RR}, 95 \% \mathrm{CI})\end{array}$ \\
\hline BAPN, 1991 [25] & SSNS & NR & $\begin{array}{l}\mathrm{L}(2.5 \mathrm{mg} / \mathrm{kg} \text { qod })+\text { steroid vs. } \\
\text { Placebo + steroid (16 weeks) }\end{array}$ & $61 / 30$ & $\begin{array}{c}\text { L } 57 \%(17 / 31) \\
\text { Placebo } 87 \%(26 / 30)\end{array}$ & 16 & $\begin{array}{c}0.63 \\
(0.45-0.90)\end{array}$ \\
\hline Weiss, $1993 *[61]$ & FRNS, SDNS & $\begin{array}{l}\text { Prior Tx with other } \\
\text { IS within } 6 \mathrm{mo}\end{array}$ & $\begin{array}{l}\mathrm{L}(2.5 \mathrm{mg} / \mathrm{kg} \text { twice a week })+\text { steroid } \\
\text { vs. Placebo + steroid }(6 \mathrm{mo})\end{array}$ & $48 / 26$ & $\begin{array}{c}\text { L } 95 \%(21 / 22) \\
\text { Placebo } 81 \%(21 / 26)\end{array}$ & 24 & $\begin{array}{c}1.18 \\
(0.96-1.46)\end{array}$ \\
\hline Dayal, 1994 [62] & SSNS & SRNS & $\begin{array}{l}\mathrm{L}(2-3 \mathrm{mg} / \mathrm{kg} \text { twice a week })+\text { steroid } \\
\text { vs. steroid alone ( } 48 \text { weeks) }\end{array}$ & $36 / 14$ & $\begin{array}{c}\text { L } 41 \%(9 / 22) \\
\text { Steroid alone } 71 \%(10 / 14)\end{array}$ & 48 & $\begin{array}{c}0.57 \\
(0.31-1.05)\end{array}$ \\
\hline Rashid, 1996 * [63] & FRNS, SDNS & NR & $\begin{array}{l}\mathrm{L}(2.5 \mathrm{mg} / \mathrm{kg} \text { qod })+\text { steroid vs. } \\
\text { steroid alone }(6 \mathrm{mo})\end{array}$ & $40 / 20$ & $\begin{array}{c}\text { L 30\% (6/20) } \\
\text { Steroid alone 60\% (12/20) }\end{array}$ & 44 & $\begin{array}{c}0.50 \\
(0.23-1.07)\end{array}$ \\
\hline Sural, $2001 *[64]$ & FRNS, SDNS & NR & $\begin{array}{l}\mathrm{L}(2.5 \mathrm{mg} / \mathrm{kg} \text { qod })+\text { steroid vs. } \\
\text { steroid alone }(6 \mathrm{mo})\end{array}$ & $58 / 28$ & $\begin{array}{c}\text { L 27\% (8/30) } \\
\text { Steroid alone } 82 \%(23 / 28)\end{array}$ & 48 & $\begin{array}{c}0.32 \\
(0.17-0.60)\end{array}$ \\
\hline Donia, 2005 [34] & SSNS & NR & $\begin{array}{l}\mathrm{L}(2.5 \mathrm{mg} / \mathrm{kg} \text { qod })+\text { steroid vs. IV } \\
\text { CPA + steroid }\end{array}$ & $40 / 20$ & $\begin{array}{c}\text { L 50\% (10/20) } \\
\text { CPA 55\% (11/20) }\end{array}$ & 96 & $\begin{array}{c}0.91 \\
(0.50-1.64)\end{array}$ \\
\hline $\begin{array}{c}\text { Al-Saran, } 2006 \\
\text { [65] }\end{array}$ & FRNS, SDNS & Prior Tx with IS & $\begin{array}{l}\mathrm{L}(2.5 \mathrm{mg} / \mathrm{kg} \text { qod })+\text { steroid vs. } \\
\text { steroid alone }(1 \mathrm{yr})\end{array}$ & $56 / 24$ & $\begin{array}{c}\text { Levamisole 9\% (3/32) } \\
\text { Steroid alone 50\% (12/24) }\end{array}$ & 48 & $\begin{array}{c}0.19 \\
(0.06-0.59) \\
\end{array}$ \\
\hline $\begin{array}{l}\text { Abeyagunawardena } \\
\left.\text { AS, 2006* }{ }^{*} 66\right]\end{array}$ & SSNS & NR & $\mathrm{L}(2.5 \mathrm{mg} / \mathrm{kg}$ qod $)+$ steroid vs. No Tx & $76 / 34$ & $\begin{array}{c}\text { L 19\% (8/42) } \\
\text { Control 76\% (26/34) }\end{array}$ & 48 & $\begin{array}{c}0.25 \\
(0.13-0.48)\end{array}$ \\
\hline Gruppen, 2018 [28] & FRNS, SDNS & $\begin{array}{l}\text { Unresponsiveness } \\
\text { to cyclosporine or } \\
\text { MMF }\end{array}$ & $\begin{array}{l}\mathrm{L}(2.5 \mathrm{mg} / \mathrm{kg} \text { qod })+\text { steroid vs. } \\
\text { Placebo (maximum } 21 \text { days) }\end{array}$ & $99 / 49$ & $\begin{array}{c}\text { L } 66 \%(33 / 50) \\
\text { Placebo } 86 \%(42 / 49)\end{array}$ & 48 & $\begin{array}{c}0.30 \\
(0.11-0.82)\end{array}$ \\
\hline Sinha, 2019 [30] & FRNS, SDNS & $\begin{array}{l}\text { Prior Tx with other } \\
\text { IS }\end{array}$ & $\mathrm{L}(2-2.5 \mathrm{mg} / \mathrm{kg}$ qod) vs. MMF & $149 / 76$ & $\begin{array}{c}\text { L 66\% (48/73) } \\
\text { MMF 59\% (45/76) }\end{array}$ & 48 & $\begin{array}{c}0.79 \\
(0.58-1.07)\end{array}$ \\
\hline
\end{tabular}


Table 3. Summary of meta-analysis of RCTs on levamisole treatment in nephrotic syndrome.

\begin{tabular}{|c|c|c|c|c|c|c|c|c|c|c|}
\hline Author, Year & Comparison & Outcome & $\begin{array}{l}\text { No. of } \\
\text { Studies }\end{array}$ & $\begin{array}{c}\text { No. of } \\
\text { Cases/Controls }\end{array}$ & $\begin{array}{l}\text { Type of } \\
\text { Metrics }\end{array}$ & $\begin{array}{c}\text { Reported } \\
\text { Summary } \\
\text { Effect }(95 \% \mathrm{CI})\end{array}$ & $\begin{array}{c}\text { Reported } \\
p \text {-Value }\end{array}$ & $\begin{array}{c}\text { Largest } \\
\text { Effect } \\
(95 \% \text { CI })\end{array}$ & $\begin{array}{c}\mathrm{I}^{2} \\
(p \text { Value })\end{array}$ & $\begin{array}{c}\text { No. of } \\
\text { Significant } \\
\text { Study/Total } \\
\text { Study }\end{array}$ \\
\hline $\begin{array}{c}\text { Durkan AM, et al. } \\
2001 \text { [67] }\end{array}$ & $\begin{array}{l}\text { L vs. } \\
\text { placebo }\end{array}$ & $\begin{array}{l}\text { Relapse } \\
(4-12 \mathrm{mo})\end{array}$ & 3 & $137 / 64$ & $\mathrm{RR}$ & $0.60(0.45-0.79)$ & 0.0004 & $\begin{array}{c}0.63 \\
(0.45-0.90)\end{array}$ & $\begin{array}{l}0.0 \% \\
(0.84)\end{array}$ & $1 / 3$ \\
\hline $\begin{array}{c}\text { Durkan AM, et al. } \\
2005 \text { [68] }\end{array}$ & $\begin{array}{c}\text { L vs. } \\
\text { placebo/no } \\
\text { treatment }\end{array}$ & $\begin{array}{l}\text { Relapse } \\
(4-12 \mathrm{mo})\end{array}$ & 4 & $185 / 90$ & $\mathrm{RR}$ & $0.71(0.41-1.23)$ & 0.23 & $\begin{array}{c}0.63 \\
(0.45-0.90)\end{array}$ & $\begin{array}{c}86 \% \\
(<0.001)\end{array}$ & $1 / 4$ \\
\hline $\begin{array}{l}\text { Hodson EM, et al. } \\
\text { 2008. [69] }\end{array}$ & $\begin{array}{l}\text { L vs. } \\
\text { placebo }\end{array}$ & $\begin{array}{c}\text { Relapse } \\
(4-12 \mathrm{mo})\end{array}$ & 6 & $317 / 148$ & $\mathrm{RR}$ & $0.50(0.25-0.99)$ & 0.046 & $\begin{array}{c}0.25 \\
(0.13-0.48)\end{array}$ & $\begin{array}{c}92 \% \\
(0.046)\end{array}$ & $3 / 6$ \\
\hline $\begin{array}{l}\text { Hodson EM, et al. } \\
2008 \text { [69] }\end{array}$ & L vs. IV CPA & Relapse (E) & 1 & $40 / 20$ & $\mathrm{RR}$ & $0.91(0.50-1.64)$ & NA & - & - & $0 / 1$ \\
\hline $\begin{array}{l}\text { Pravitsitthikul N, } \\
\text { et al. } 2013 \text { [31] }\end{array}$ & $\begin{array}{l}\text { L vs. } \\
\text { steroids or } \\
\text { placebo or } \\
\text { both, or no } \\
\text { treatment }\end{array}$ & $\begin{array}{l}\text { Relapse } \\
(4-12 \mathrm{mo})\end{array}$ & 7 & $375 / 176$ & RR & $0.47(0.24-0.89)$ & 0.021 & $\begin{array}{c}0.25 \\
(0.13-0.48)\end{array}$ & $\begin{array}{c}92 \% \\
(<0.001)\end{array}$ & $4 / 7$ \\
\hline $\begin{array}{l}\text { Pravitsitthikul N, } \\
\text { et al. } 2013 \text { [31] }\end{array}$ & L vs. IV CPA & Relapse (E) & 2 & $97 / 47$ & $\mathrm{RR}$ & $2.14(0.22-20.95)$ & 0.51 & $\begin{array}{c}7.20 \\
(0.96-53.89)\end{array}$ & $\begin{array}{c}79 \% \\
(0.03)\end{array}$ & $0 / 2$ \\
\hline
\end{tabular}

CPA: Cyclophosphamide, E: end of therapy, L: Levamisole, mo: months, NA: Significant, RCT: randomized controlled trial, RR: relative risk. Largest effect: Effect size (95\% CI) and $p$-value from the largest study in each meta-analysis. 


\section{Known Side Effects of Levamisole Treatment}

Levamisole is a U.S. Food and Drug Administration pregnancy category C drug. It was withdrawn in the United States and Europe because of adverse effects (agranulocytosis and the risk of developing anti-neutrophil cytoplasmic antibody (ANCA)-positive vasculitis) and lack of clear indications [70]. However, the adverse effects of levamisole are mostly mild and transient, disappearing after its discontinuation. Common adverse effects include gastrointestinal symptoms (nausea, abdominal cramps), and pyrexia [71]. There were only a few serious adverse events documented in studies focusing on iNS. Single cases of gastrointestinal disturbances [31,41] or leukopenia $[25,31]$ have been reported in the RCTs, probably caused by the use of levamisole. Most retrospective studies reported no side effects of levamisole [37,39,40], or only minor reversible effects such as rash, fever, abdominal pain, elevated liver enzymes, neutropenia or thrombocytopenia, which all disappeared after cessation of levamisole [36,52]. In their placebo-controlled multi-centric trial, Gruppen et al. showed that the use of levamisole was safe [28]. However, the definition of leukopenia was different in each study. In addition, recent studies have reported adverse events as neutropenia instead of leukopenia. Therefore, there is a clear limitation related to reporting all reported adverse effects together. Nevertheless, a comprehensive examination of reported studies indicates that the incidence of leukopenia varies from 0 to $20 \%$. Of the 1391 cases that reported adverse effects of levamisole, 51 cases (leukopenia or neutropenia) had leukopenia (3.7\%) (Table 4). This seems not much different from the incidence reported in other diseases [72]. Leukopenia related to the use of levamisole was spontaneously reversible after discontinuation of the treatment [28]. Levamisole can cause a variety of dermatologically adverse effects. It can cause lichenoid eruption, fixed dug eruptions, leg ulcers, purpura of the ears, and cutaneous necrosis [72]. Like other adverse events, symptoms resolved when levamisole treatment was stopped. In addition, leukoencephalopathy, skin necrosis, hyponatremia, acute coronary syndrome, pulmonary hypertension, granulomatosis with polyangiitis (GPA), and pyoderma gangrenosum were reported in single cases [73]. As levamisole is adulterated with cocaine, it may be used on the "black market" to enhance cocaine effects. The frequency of levamisole-induced GPA is increasing in the literature, with the proposed mechanisms having been recently highlighted [74].

Table 4. Reported side effects of levamisole in nephrotic syndrome.

\begin{tabular}{|c|c|c|c|c|c|c|c|}
\hline Author, Year & Levamisole Dose & Number & Leukopenia & $\begin{array}{c}\text { GI } \\
\text { Upset }\end{array}$ & $\begin{array}{l}\text { Skin } \\
\text { Rash }\end{array}$ & Arthritis & Other \\
\hline BAPN, 1991 [25] & $2.5 \mathrm{mg} / \mathrm{kg}$ qod & 31 & 0 & 1 & 0 & 0 & 0 \\
\hline Weiss, $1993 *[61]$ & $2.5 \mathrm{mg} / \mathrm{kg}$ twice a week & 22 & 0 & 0 & 0 & 0 & 0 \\
\hline Sural, $2001 *[64]$ & $2.5 \mathrm{mg} / \mathrm{kg}$ qod & 30 & 1 & 0 & 0 & 0 & 0 \\
\hline Donia, 2005 [34] & $2.5 \mathrm{mg} / \mathrm{kg}$ qod & 20 & 0 & 0 & 0 & 0 & $14^{\mathrm{a}}$ \\
\hline Al-Saran, 2006 [65] & $2.5 \mathrm{mg} / \mathrm{kg}$ qod & 32 & 0 & 1 & 0 & 0 & 0 \\
\hline Gruppen, 2018 [28] & $2.5 \mathrm{mg} / \mathrm{kg}$ qod & 50 & 8 & 1 & 0 & 1 & $26^{b}$ \\
\hline Tanphaichitr P, 1980 [22] & $1.5-3.9 \mathrm{mg} / \mathrm{kg}$ twice a week & 7 & 0 & 0 & 0 & 0 & 0 \\
\hline Niaudet P, 1984 [42] & $2.5 \mathrm{mg} / \mathrm{kg}$ twice a week & 30 & 7 & 0 & 0 & 0 & 0 \\
\hline Metha KP, 1986 [43] & $2.5 \mathrm{mg} / \mathrm{kg}$ qod & 14 & 0 & 3 & 1 & 0 & $2^{c}$ \\
\hline La Manna A, 1988 [44] & $2.5 \mathrm{mg} / \mathrm{kg}$ qd & 13 & 0 & 1 & 1 & 0 & $1^{\mathrm{d}}$ \\
\hline Prandota J, 1989 [75] & $2.1-3.1 \mathrm{mg} / \mathrm{kg}$ qd & 6 & 0 & 0 & 0 & 0 & $6^{\mathrm{e}}$ \\
\hline Srivastava RN, 1991 [45] & $5 \mathrm{mg} / \mathrm{kg}$ qod & 12 & 1 & 0 & 1 & 0 & 0 \\
\hline Alsaran K, 2001 [50] & $2.5 \mathrm{mg} / \mathrm{kg}$ qod & 24 & 0 & 0 & 1 & 0 & 0 \\
\hline Donia AF, 2002 [51] & $2.5 \mathrm{mg} / \mathrm{kg}$ qod & 20 & 0 & 0 & 0 & 0 & 0 \\
\hline Al-Ibrahim AA, 2003 [52] & $2.5 \mathrm{mg} / \mathrm{kg}$ qod & 24 & 1 & 2 & 2 & 0 & 0 \\
\hline Sumegi V, 2004 [53] & $2 \mathrm{mg} / \mathrm{kg}$ qd & 34 & 5 & 0 & 0 & 0 & 0 \\
\hline Fu LS, 2004 [54] & $2-3 \mathrm{mg} / \mathrm{kg}$ qd or qod & 36 & 9 & 0 & 0 & 0 & 0 \\
\hline Hafeez F, 2006 [55] & $2.5 \mathrm{mg} / \mathrm{kg}$ qod & 70 & 0 & 0 & 0 & 0 & 0 \\
\hline Boyer O, 2008 [37] & $2.5 \mathrm{mg} / \mathrm{kg}$ twice a week & 10 & 0 & 0 & 0 & 0 & 0 \\
\hline Madani A, 2010 [38] & $2.5 \mathrm{mg} / \mathrm{kg}$ qod & 304 & 1 & 0 & 0 & 0 & $1^{f}$ \\
\hline Elmas AT, 2013 [40] & $2.5 \mathrm{mg} / \mathrm{kg}$ twice a week & 29 & 0 & 0 & 0 & 0 & 0 \\
\hline
\end{tabular}


Table 4. Cont.

\begin{tabular}{|c|c|c|c|c|c|c|c|}
\hline Author, Year & Levamisole Dose & Number & Leukopenia & $\begin{array}{c}\text { GI } \\
\text { Upset }\end{array}$ & $\begin{array}{l}\text { Skin } \\
\text { Rash }\end{array}$ & Arthritis & Other \\
\hline Ekambaram S, 2014 [35] & $2 \mathrm{mg} / \mathrm{kg}$ qd & 97 & 0 & 0 & 0 & 0 & 0 \\
\hline Jiang L, 2015 [58] & $1.25 \mathrm{mg} / \mathrm{kg}$ qod & 15 & 3 & 2 & 2 & 0 & 0 \\
\hline Kuzma-Mroczkowska,2016 [36] & $2.5 \mathrm{mg} / \mathrm{kg}$ qod & 53 & 1 & 3 & 9 & 0 & $6^{g}$ \\
\hline Alsaran K, 2017 [59] & $2.5 \mathrm{mg} / \mathrm{kg}$ qod & 20 & 4 & 0 & 0 & 0 & 0 \\
\hline Abeyagunawardena AS, 2017 [60] & $2.5 \mathrm{mg} / \mathrm{kg} \mathrm{qd}$ and qod & 58 & 0 & 0 & 0 & 0 & 0 \\
\hline Basu B, 2017 [39] & $2.5 \mathrm{mg} / \mathrm{kg} \mathrm{qod}$ & 129 & 0 & 0 & 0 & 0 & $3^{h}$ \\
\hline Youssef DM, 2018 [76] & $2.5 \mathrm{mg} / \mathrm{kg}$ qod & 23 & 0 & 0 & 0 & 0 & 0 \\
\hline Total & & 1391 & $\begin{array}{c}51 \\
(3.7 \%)\end{array}$ & $\begin{array}{c}33 \\
(2.4 \%)\end{array}$ & $\begin{array}{c}21 \\
(1.5 \%)\end{array}$ & $\begin{array}{c}1 \\
(0 \%)\end{array}$ & $\begin{array}{c}59 \\
(4.2 \%)\end{array}$ \\
\hline
\end{tabular}

BAPN: British Association for Pediatric Nephrology, GI: gastrointestinal, qd: once daily, qod: every other day. a Respiratory infection (9), Scalp infection (2), Urinary tract infection (1), sialadenitis (1), personality change (1). $\mathrm{b}$ Cough (6), Nasopharyngitis (8), Pyrexia (10), ANCA-associated vasculitis (1), Reduced glomerular filtration rate (GFR) (1). ${ }^{c}$ Transient hematuria (2). d Allergic conjunctivitis (1). e Thrombocytopenia (4), elevated liver transaminases (2). ${ }^{\mathrm{f}}$ Vertigo (1). ${ }^{\mathrm{g}}$ Elevated liver transaminases (3), arthralgia (2), thrombocytopenia (1). ${ }^{\mathrm{h}}$ Malaria (2), transient mood change (1). * Data were extracted from the abstract.

Recent studies showed almost the same effect in adults with iNS [58]. Both KDIGO and Cochrane recommend levamisole with evidence grade $1 \mathrm{~b}$ as an alternative medication to reduce the steroid load of patients with frequently relapsing iNS $[69,77]$. However, the precise pharmaceutical mechanism of this drug still needs to be evaluated. In addition, levamisole is used at a similar dose $(2.0-2.5 \mathrm{mg} / \mathrm{kg}$ on alternate days, maximum $150 \mathrm{mg}$ ) to treat other diseases in adults [20,21,72], but few studies have investigated its efficacy in the management of nephrotic syndrome.

\section{Mechanism Hypothesis}

Proteinuria in nephrotic syndrome has been linked to various mechanistic dysfunctions in podocytes, leading to podocyte foot process effacement. MCNS is a pathological condition, which is characterized by subtle glomerular lesions causing massive and reversible proteinuria that is usually steroid sensitive. Glucocorticoids have historically been used as a first-line therapy in MCNS on the basis of their immunosuppressive function. However, it has been shown that podocytes serve as non-immunologic targets for several immunosuppressive drugs like cyclosporine and glucocorticoids [78]. Th2 cytokines (Interleukin (IL)-13, IL-4, IL-5) are increased in patients with nephrotic syndrome, and changes in vascular permeability have been reported, which are both involved in the complex pathogenesis of proteinuria in nephrotic syndrome [3,79-81]. Lai et al. showed that IL-13 transfected rats showed increased levels of proteinuria, raised serum cholesterol and decreased serum albumin levels and podocyte foot processes showed fusion mimicking MCNS [82]. The podocyte's actin cytoskeleton is a discovered target for newer therapies. Calcineurin inhibitors, for example, block the dephosphorylation of the cytoskeleton component synaptopodin, and steroids increase actin polymerization and stability $[83,84]$.

Levamisole is a synthetic imidazole derivative, which was used for its anthelmintic effects by acting as a nicotinic acetylcholine receptor agonist. Because various adverse effects including severe agranulocytosis in animals have been reported, levamisole is no longer used to treat any parasitic infections. Levamisole increases lymphocyte cyclic guanosine monophosphate (cGMP) by affecting cholinergic activity on $\mathrm{T}$ lymphocyte. Increased adenosine deaminase and free radicals act on both $\mathrm{T}$ and B lymphocytes. However, this drug has an additional immune-modulating action. It induces type 1 (Th1) and type 2 (Th2) immune responses through enhancing IL-18 activity [3]. It is also known that an enhanced Th1 or Th2 immune response is associated with several inflammatory and autoimmune diseases, such as SLE [85], asthma [86,87] and type 1 diabetes [88]. Therefore, levamisole is thought to have immunomodulatory potential. Szeto and colleagues showed that treatment with levamisole leads to a shift in the immune response in Brown Norway rats. This caused the augmentation of Th1 response and reduced the Th2 response [89]. Its effect is more pronounced on T lymphocyte than on B lymphocytes. Increased $\mathrm{CD} 4^{+} \mathrm{T} / \mathrm{CD} 8^{+} \mathrm{T}$ Cell ratio and reduced IL-4 have been observed 
in levamisole-treated lichen planus patients and in a mouse model $[87,90]$. Experimental studies have shown that in mice with allergic rhinitis levamisole decreased IL-4, IL-5, and IL-13 mRNA and at the same time enhanced the expression of IL-12, IL-18 and interferon (IFN)-gamma mRNA [71]. IL-13 is an important Th2 cytokine involved in the induction of MCNS in a rat experiment model by the down-regulation of nephrin, podocin, and dystroglycan. It can also up-regulate B7-1 [65], and is altering the expression of zonula occludens-1 (ZO-1) in human podocytes [91]. In summary, the beneficial effect of levamisole on MCNS might be due to its suppressing effect on IL-13 expression. Upregulation of T helper-1 (Th1) cells and down-regulation of Th2 cells is considered to be the main mechanism for treating FRNS or SDNS patients $[30,90,92]$. Levamisole reduces immunoglobulin (IG) G, IG M, and circulating immune complex, which inhibit B cell activity [93]. Activated B cells caused podocyte foot process effacement via IL-4 [81]. In addition, total B cell increased in pediatric SSNS patients [94]. Although more mechanisms are still needed, not only T cells, but also B cells are considered to be therapeutic targets of levamisole.

Recent work has shown an additional direct effect of levamisole on podocytes. In an immortalized human podocyte culture, the authors were able to show that levamisole enhanced the expression and activity of the glucocorticoid receptor (GR). GR is present in glomerular cells including podocytes. Furthermore, levamisole protected podocytes in a puromycin aminonucleoside (PAN)-treated cell model. In this model, the effectiveness of levamisole is blocked by the GR antagonist mifepristone (RU486), suggesting that GR signaling is a critical target of levamisole's action. Activated GR restoration of Bcl-2 and reduction of p53 in PAN treated podocytes resulting in inhibition of podocyte apoptosis [95]. Another study has shown that activated GR upregulates the expression of nephrin and Rho-A, leading to enhanced actin filament stability [96,97]. These data indicate that levamisole might be effective in nephrotic syndrome in adults as well as in children [58]. Those effects of levamisole are presented in Figure 1.

In summary, there is no universally accepted explanation how levamisole works in SSNS. Levamisole-induced changes in the immune-mediated response may cause reduction of relapses in predominantly immune mediated diseases. This may be one explanation for its ineffectiveness in cases of steroid-resistant nephrotic syndrome [98].

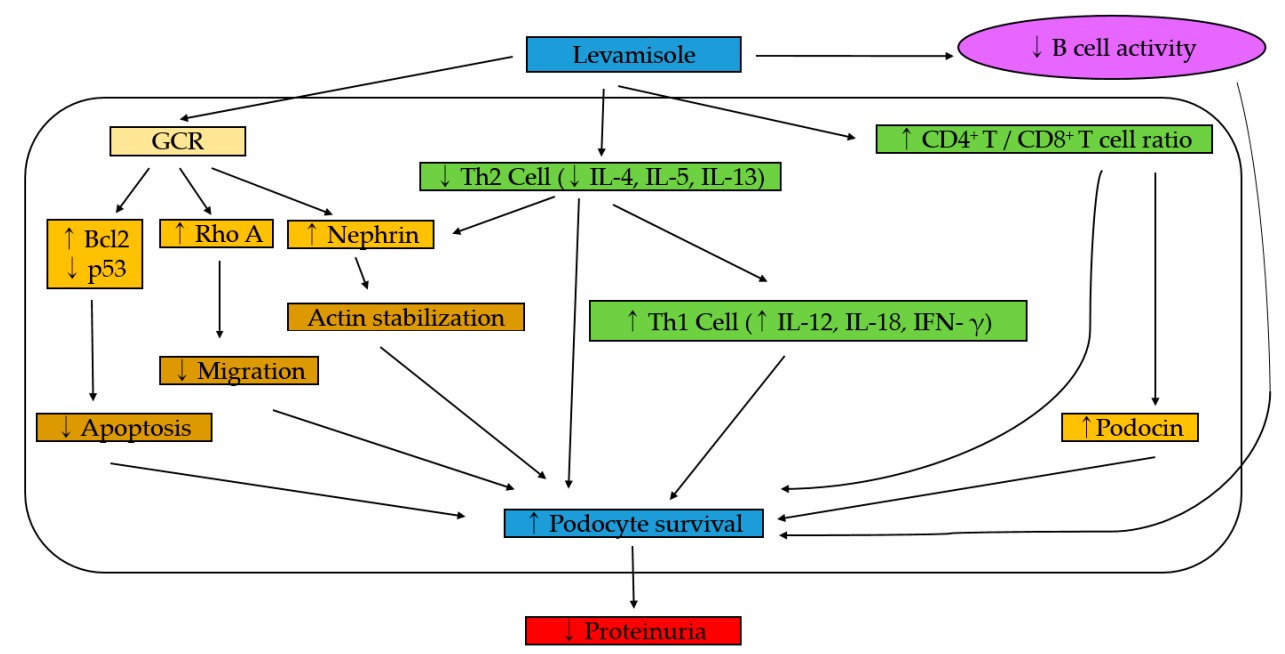

Figure 1. The summary effects of levamisole in iNS. Bcl2: B-cell lymphoma 2, CD: Cluster of differentiation, IFN- $\gamma$ : interferon gamma, IL: interleukin, iNS: idiopathic nephrotic syndrome, GCR: glucocorticoid receptor, p53: tumor protein p53, Th Cell: The T helper cells.

\section{Summary and Conclusions}

The effect of immunosuppressive agents in proteinuric glomerular diseases is attributed to immune-dependent mechanisms. However, recent studies have revealed that the anti-proteinuric effect of commonly used drugs might be related not only to their immunosuppressive actions, but 
also occur via a direct effect on podocytes. This also includes levamisole. Levamisole seems to be effective in reducing the risk of disease relapses in steroid sensitive forms of NS. In the reported studies, few serious side effects have been reported, compared to other steroid-sparing agents. There are hints that it is less effective compared to cyclophosphamide [34,69] and compared to MMF [39], but in comparison to both of these drugs, the adverse effects spectrum is beneficial. However, RCTs comparing different steroid-sparing agents head to head with levamisole in the treatment of SSNS are still lacking. Given the low frequency of side effects and that most adverse events improve with discontinuation of levamisole and KDIGO recommendations [77], levamisole could be a valuable alternative therapy for patients having frequent relapsing iNS. In addition, it has been shown that even after discontinuation of levamisole, long-term remission can be achieved. Therefore, levamisole should be considered a precious option in the treatment of SSNS, especially in cases of contraindications for immunosuppressive drugs. Regular monitoring of white blood count cells, renal function and testing of ANCA is advised in cases reporting symptoms of vasculitis.

Author Contributions: J.I.S. and A.K.M. designed the study, J.Y.L. collected the data and did the analysis. J.Y.L., A.K.M., M.J.K., A.K., J.I.S., J.W.Y. and J.O. wrote the first draft of the manuscript and gave critical comments on manuscript draft. All authors had full access to all the study data. All authors reviewed, wrote and approved the final version. The corresponding author had final responsibility for the decision to submit for publication.

Conflicts of Interest: The authors declare no conflict of interest.

\section{References}

1. Eddy, A.A.; Symons, J.M. Nephrotic syndrome in childhood. Lancet 2003, 362, 629-639. [CrossRef]

2. Kemper, M.J.; Zepf, K.; Klaassen, I.; Link, A.; Muller-Wiefel, D.E. Changes of lymphocyte populations in pediatric steroid-sensitive nephrotic syndrome are more pronounced in remission than in relapse. Am. J. Nephrol. 2005, 25, 132-137. [CrossRef] [PubMed]

3. Shalaby, S.A.; Al-Edressi, H.M.; El-Tarhouny, S.A.; Fath El-Bab, M.; Zolaly, M.A. Type 1/type 2 cytokine serum levels and role of interleukin-18 in children with steroid-sensitive nephrotic syndrome. Arab. J. Nephrol. Transpl. 2013, 6, 83-88.

4. Greka, A.; Mundel, P. Cell biology and pathology of podocytes. Annu. Rev. Physiol. 2012, 74, 299-323. [CrossRef] [PubMed]

5. Van Husen, M.; Kemper, M.J. New therapies in steroid-sensitive and steroid-resistant idiopathic nephrotic syndrome. Pediatr. Nephrol. 2011, 26, 881-892. [CrossRef] [PubMed]

6. Sureshkumar, P.; Hodson, E.M.; Willis, N.S.; Barzi, F.; Craig, J.C. Predictors of remission and relapse in idiopathic nephrotic syndrome: A prospective cohort study. Pediatr. Nephrol. 2014, 29, 1039-1046. [CrossRef] [PubMed]

7. Ruth, E.M.; Kemper, M.J.; Leumann, E.P.; Laube, G.F.; Neuhaus, T.J. Children with steroid-sensitive nephrotic syndrome come of age: Long-term outcome. J. Pediatr. 2005, 147, 202-207. [CrossRef]

8. The primary nephrotic syndrome in children. Identification of patients with minimal change nephrotic syndrome from initial response to prednisone. A report of the International Study of Kidney Disease in Children. J. Pediatr. 1981, 98, 561-564. [CrossRef]

9. Arbeitsgemeinschaft für Pädiatrische Nephrologie. Short versus standard prednisone therapy for initial treatment of idiopathic nephrotic syndrome in children. Lancet 1988, 1, 380-383. [CrossRef]

10. Ehrich, J.H.; Brodehl, J. Long versus standard prednisone therapy for initial treatment of idiopathic nephrotic syndrome in children. Eur. J. Pediatr. 1993, 152, 357-361. [CrossRef]

11. Tarshish, P.; Tobin, J.N.; Bernstein, J.; Edelmann, C.M., Jr. Prognostic significance of the early course of minimal change nephrotic syndrome: Report of the International Study of Kidney Disease in Children. J. Am. Soc. Nephrol. 1997, 8, 769-776. [PubMed]

12. Kyrieleis, H.A.; Lowik, M.M.; Pronk, I.; Cruysberg, H.R.; Kremer, J.A.; Oyen, W.J.; van den Heuvel, B.L.; Wetzels, J.F.; Levtchenko, E.N. Long-term outcome of biopsy-proven, frequently relapsing minimal-change nephrotic syndrome in children. Clin. J. Am. Soc. Nephrol. 2009, 4, 1593-1600. [CrossRef] [PubMed]

13. Poetker, D.M.; Reh, D.D. A comprehensive review of the adverse effects of systemic corticosteroids. Otolaryngol. Clin. N. Am. 2010, 43, 753-768. [CrossRef] [PubMed] 
14. Fardet, L.; Cabane, J.; Lebbe, C.; Morel, P.; Flahault, A. Incidence and risk factors for corticosteroid-induced lipodystrophy: A prospective study. J. Am. Acad. Derm. 2007, 57, 604-609. [CrossRef] [PubMed]

15. Larkins, N.; Kim, S.; Craig, J.; Hodson, E. Steroid-sensitive nephrotic syndrome: An evidence-based update of immunosuppressive treatment in children. Arch. Dis. Child. 2016, 101, 404-408. [CrossRef] [PubMed]

16. Martin, F.; Lauwerys, B.; Lefebvre, C.; Devogelaer, J.P.; Houssiau, F.A. Side-effects of intravenous cyclophosphamide pulse therapy. Lupus 1997, 6, 254-257. [CrossRef] [PubMed]

17. Tedesco, D.; Haragsim, L. Cyclosporine: A review. J. Transpl. 2012, 2012, 230386. [CrossRef] [PubMed]

18. Kitchin, J.E.; Pomeranz, M.K.; Pak, G.; Washenik, K.; Shupack, J.L. Rediscovering mycophenolic acid: A review of its mechanism, side effects, and potential uses. J. Am. Acad. Derm. 1997, 37, 445-449. [CrossRef]

19. Mihatsch, M.J.; Kyo, M.; Morozumi, K.; Yamaguchi, Y.; Nickeleit, V.; Ryffel, B. The side-effects of ciclosporine-A and tacrolimus. Clin. Nephrol. 1998, 49, 356-363.

20. Kar, H.K.; Bhatia, V.N.; Kumar, C.H.; Sirumban, P.; Roy, R.G. Evaluation of levamisole, an immunopotentiator, in the treatment of lepromatous leprosy. Indian J. Lepr. 1986, 58, 592-600.

21. Mutch, R.S.; Hutson, P.R. Levamisole in the adjuvant treatment of colon cancer. Clin. Pharm. 1991, 10, 95-109. [CrossRef] [PubMed]

22. Tanphaichitr, P.; Tanphaichitr, D.; Sureeratanan, J.; Chatasingh, S. Treatment of nephrotic syndrome with levamisole. J. Pediatr. 1980, 96, 490-493. [CrossRef]

23. Bagga, A.; Sharma, A.; Srivastava, R.N. Levamisole therapy in corticosteroid-dependent nephrotic syndrome. Pediatr. Nephrol. 1997, 11, 415-417. [CrossRef] [PubMed]

24. Neuhaus, T.J.; Fay, J.; Dillon, M.J.; Trompeter, R.S.; Barratt, T.M. Alternative treatment to corticosteroids in steroid sensitive idiopathic nephrotic syndrome. Arch. Dis. Child. 1994, 71, 522-526. [CrossRef] [PubMed]

25. British Association for Paediatric Nephrology. Levamisole for corticosteroid-dependent nephrotic syndrome in childhood. Lancet 1991, 337, 1555-1557. [CrossRef]

26. Kemper, M.J.; Amon, O.; Timmermann, K.; Altrogge, H.; Muller-Wiefel, D.E. The treatment with levamisole of frequently recurring steroid-sensitive idiopathic nephrotic syndrome in children. Dtsch. Med. Wochenschr. 1998, 123, 239-243. [CrossRef] [PubMed]

27. Bagga, A.; Ali, U.; Banerjee, S.; Kanitkar, M.; Phadke, K.D.; Senguttuvan, P.; Sethi, S.; Shah, M. Management of steroid sensitive nephrotic syndrome: Revised guidelines. Indian Pediatr. 2008, 45, 203-214. [CrossRef]

28. Gruppen, M.P.; Bouts, A.H.; Jansen-van der Weide, M.C.; Merkus, M.P.; Zurowska, A.; Maternik, M.; Massella, L.; Emma, F.; Niaudet, P.; Cornelissen, E.A.M.; et al. A randomized clinical trial indicates that levamisole increases the time to relapse in children with steroid-sensitive idiopathic nephrotic syndrome. Kidney Int. 2018, 93, 510-518. [CrossRef]

29. Kemper, M.J.; Neuhaus, T.J. Levamisole in relapsing steroid-sensitive nephrotic syndrome: Where do we stand? Kidney Int. 2018, 93, 310-313. [CrossRef]

30. Sinha, A.; Puraswani, M.; Kalaivani, M.; Goyal, P.; Hari, P.; Bagga, A. Efficacy and safety of mycophenolate mofetil versus levamisole in frequently relapsing nephrotic syndrome: An open-label randomized controlled trial. Kidney Int. 2019, 95, 210-218. [CrossRef]

31. Pravitsitthikul, N.; Willis, N.S.; Hodson, E.M.; Craig, J.C. Non-corticosteroid immunosuppressive medications for steroid-sensitive nephrotic syndrome in children. Cochrane Database Syst. Rev. 2013, Cd002290. [CrossRef] [PubMed]

32. Hodson, E.M.; Craig, J.C.; Willis, N.S. Evidence-based management of steroid-sensitive nephrotic syndrome. Pediatr. Nephrol. 2005, 20, 1523-1530. [CrossRef] [PubMed]

33. Davin, J.C.; Merkus, M.P. Levamisole in steroid-sensitive nephrotic syndrome of childhood: The lost paradise? Pediatr. Nephrol. 2005, 20, 10-14. [CrossRef] [PubMed]

34. Donia, A.F.; Ammar, H.M.; El-Agroudy Ael, B.; Moustafa Fel, H.; Sobh, M.A. Long-term results of two unconventional agents in steroid-dependent nephrotic children. Pediatr. Nephrol. 2005, 20, 1420-1425. [CrossRef] [PubMed]

35. Ekambaram, S.; Mahalingam, V.; Nageswaran, P.; Udani, A.; Geminiganesan, S.; Priyadarshini, S. Efficacy of levamisole in children with frequently relapsing and steroid-dependent nephrotic syndrome. Indian Pediatr. 2014, 51, 371-373. [CrossRef] [PubMed]

36. Kuzma-Mroczkowska, E.; Skrzypczyk, P.; Panczyk-Tomaszewska, M. Levamisole therapy in children with frequently relapsing and steroid-dependent nephrotic syndrome: A single-center experience. Cent. Eur. J. Immunol. 2016, 41, 243-247. [CrossRef] [PubMed] 
37. Boyer, O.; Moulder, J.K.; Grandin, L.; Somers, M.J. Short- and long-term efficacy of levamisole as adjunctive therapy in childhood nephrotic syndrome. Pediatr. Nephrol. 2008, 23, 575-580. [CrossRef]

38. Madani, A.; Isfahani, S.T.; Rahimzadeh, N.; Fereshtehnejad, S.M.; Hoseini, R.; Moghtaderi, M.; Mohseni, P.; Ataiee, N. Effect of levamisole in steroid-dependent nephrotic syndrome. Iran. J. Kidney Dis. 2010, 4, $292-296$.

39. Basu, B.; Babu, B.G.; Mahapatra, T.K. Long-term efficacy and safety of common steroid-sparing agents in idiopathic nephrotic children. Clin. Exp. Nephrol. 2017, 21, 143-151. [CrossRef]

40. Elmas, A.T.; Tabel, Y.; Elmas, O.N. Short- and long-term efficacy of levamisole in children with steroid-sensitive nephrotic syndrome. Int. Urol. Nephrol. 2013, 45, 1047-1055. [CrossRef]

41. Kreeftmeijer-Vegter, A.R.; Dorlo, T.P.; Gruppen, M.P.; de Boer, A.; de Vries, P.J. Population pharmacokinetics of levamisole in children with steroid-sensitive nephrotic syndrome. Br. J. Clin. Pharm. 2015, 80, 242-252. [CrossRef] [PubMed]

42. Niaudet, P.; Drachman, R.; Gagnadoux, M.F.; Broyer, M. Treatment of idiopathic nephrotic syndrome with levamisole. Acta Paediatr. Scand. 1984, 73, 637-641. [CrossRef] [PubMed]

43. Mehta, K.P.; Ali, U.; Kutty, M.; Kolhatkar, U. Immunoregulatory treatment for minimal change nephrotic syndrome. Arch. Dis. Child. 1986, 61, 153-158. [CrossRef] [PubMed]

44. La Manna, A.; Polito, C.; Del Gado, R.; Foglia, A.C. Levamisole in children's idiopathic nephrotic syndrome. Child. Nephrol. Urol. 1988, 9, 200-202. [PubMed]

45. Srivastava, R.N.; Vasudev, A.S.; Bagga, A. Levamisole in nephrotic syndrome. Lancet 1991, $338,1275$. [CrossRef]

46. Meregalli, P.; Bianchetti, M.G.; Imoberdorf, G.; Lutschg, J.; Reymond, D.; Oetliker, O.H. Levamisole in children with frequently recurring idiopathic nephrotic syndrome. Schweiz. Med. Wochenschr. 1994, 124, 801-805.

47. Ksiazek, J.; Krynski, J. Evaluation of the efficacy of levamisole in corticosteroid-dependent nephrotic syndrome in children. Pediatr. Pol. 1995, 70, 1037-1042.

48. Ginevri, F.; Trivelli, A.; Ciardi, M.R.; Ghiggeri, G.M.; Parfumo, F.; Gusmano, R. Protracted levamisole in children with frequent-relapse nephrotic syndrome. Pediatr. Nephrol. 1996, 10, 550.

49. Fu, L.S.; Chi, C.S. Levamisole in steroid-sensitive nephrotic syndrome children with steroid-dependency and/or frequent relapses. Acta Paediatr. Taiwan 2000, 41, 80-84.

50. Alsaran, K.; Grisaru, S.; Stephens, D.; Arbus, G. Levamisole vs. cyclophosphamide for frequently-relapsing steroid-dependent nephrotic syndrome. Clin. Nephrol. 2001, 56, 289-294.

51. Donia, A.F.; Amer, G.M.; Ahmed, H.A.; Gazareen, S.H.; Moustafa, F.E.; Shoeib, A.A.; Ismail, A.M.; Khamis, S.; Sobh, M.A. Levamisole: Adjunctive therapy in steroid dependent minimal change nephrotic children. Pediatr. Nephrol. 2002, 17, 355-358. [CrossRef] [PubMed]

52. Al-Ibrahim, A.A.; Al-Kharraz, S.M.; Al-Sadoon, D.M.; Al-Madani, A.J.; Al-Musallam, S.A. Levamisole Therapy as a Second-line Immunosuppressive Agent in Corticosteroid-sensitive Nephrotic Syndrome in Children. Saudi J. Kidney Dis. Transpl. 2003, 14, 153-157. [PubMed]

53. Sumegi, V.; Haszon, I.; Ivanyi, B.; Bereczki, C.; Papp, F.; Turi, S. Long-term effects of levamisole treatment in childhood nephrotic syndrome. Pediatr. Nephrol. 2004, 19, 1354-1360. [CrossRef] [PubMed]

54. Fu, L.S.; Shien, C.Y.; Chi, C.S. Levamisole in steroid-sensitive nephrotic syndrome children with frequent relapses and/or steroid dependency: Comparison of daily and every-other-day usage. Nephron Clin. Pract. 2004, 97, c137-c141. [CrossRef] [PubMed]

55. Hafeez, F.; Ahmed, T.M.; Samina, U. Levamisole in steroid dependent and frequently relapsing nephrotic syndrome. J. Coll. Physicians Surg. Pak. 2006, 16, 35-37. [PubMed]

56. Chen, S.Y.; Wu, C.Y.; Tsai, I.J.; Tsau, Y.K. Treatment course of steroid-dependent nephrotic syndrome: Emphasized on treatment effect. Nephrology 2010, 15, 336-339. [CrossRef] [PubMed]

57. Skrzypczyk, P.; Panczyk-Tomaszewska, M.; Roszkowska-Blaim, M.; Wawer, Z.; Bienias, B.; Zajgzkowska, M.; Kilis-Pstrusinska, K.; Jakubowska, A.; Szczepaniak, M.; Pawlak-Bratkowska, M.; et al. Long-term outcomes in idiopathic nephrotic syndrome: From childhood to adulthood. Clin. Nephrol. 2014, 81, 166-173. [CrossRef]

58. Jiang, L.; Dasgupta, I.; Hurcombe, J.A.; Colyer, H.F.; Mathieson, P.W.; Welsh, G.I. Levamisole in steroid-sensitive nephrotic syndrome: Usefulness in adult patients and laboratory insights into mechanisms of action via direct action on the kidney podocyte. Clin. Sci. 2015, 128, 883-893. [CrossRef] [PubMed] 
59. Alsaran, K.; Mirza, K.; Al-Talhi, A.; Al-Kanani, E. Experience with second line drugs in frequently relapsing and steroid dependent childhood nephrotic syndrome in a large Saudi center. Int. J. Pediatr. Adolesc. Med. 2017, 4, 66-70. [CrossRef]

60. Abeyagunawardena, A.S.; Karunadasa, U.; Jayaweera, H.; Thalgahagoda, S.; Tennakoon, S.; Abeyagunawardena, S. Efficacy of higher-dose levamisole in maintaining remission in steroid-dependant nephrotic syndrome. Pediatr. Nephrol. 2017, 32, 1363-1367. [CrossRef]

61. Weiss, R. Randomized double-blind placebo controlled, multi-center trial of levamisole for children with frequently relapsing/steroid dependent nephrotic syndrome. J. Am. Soc. Nephrol. 1993, 4, 289.

62. Dayal, U.; Dayal, A.K.; Shastry, J.C.; Raghupathy, P. Use of levamisole in maintaining remission in steroid-sensitive nephrotic syndrome in children. Nephron 1994, 66, 408-412. [CrossRef] [PubMed]

63. Rashid, H.U.; Ahmed, S.; Fatima, N.; Khanam, A. Levamisole in the treatment of steroid dependent or frequent relapsing nephrotic syndrome in children. Bangladesh Ren. J. 1996, 15, 6-8.

64. Sural, S.; Pahari, D.K.; Mitra, K.; Bhattacharya, S.; Mondal, S.; Taraphder, A. Efficacy of levamisole compared to cyclophosphamide and steroid in frequently relapsing minimal change nephrotic syndrome. J. Am. Soc. Nephrol. 2001, 12, 126.

65. Al-Saran, K.; Mirza, K.; Al-Ghanam, G.; Abdelkarim, M. Experience with levamisole in frequently relapsing, steroid-dependent nephrotic syndrome. Pediatr. Nephrol. 2006, 21, 201-205. [CrossRef] [PubMed]

66. Abeyagunawardena, A.; Trompeter, R.S. Efficacy of levamisole as a single agent in maintaining remission in steroid dependent nephrotic syndrome. Pediatr. Nephrol. 2006, 21, 1503.

67. Durkan, A.M.; Hodson, E.M.; Willis, N.S.; Craig, J.C. Immunosuppressive agents in childhood nephrotic syndrome: A meta-analysis of randomized controlled trials. Kidney Int. 2001, 59, 1919-1927. [CrossRef]

68. Durkan, A.; Hodson, E.M.; Willis, N.S.; Craig, J.C. Non-corticosteroid treatment for nephrotic syndrome in children. Cochrane Database Syst. Rev. 2005, Cd002290. [CrossRef]

69. Hodson, E.M.; Willis, N.S.; Craig, J.C. Non-corticosteroid treatment for nephrotic syndrome in children. Cochrane Database Syst. Rev. 2008, Cd002290. [CrossRef]

70. Cascio, M.J.; Jen, K.Y. Cocaine/levamisole-associated autoimmune syndrome: A disease of neutrophil-mediated autoimmunity. Curr. Opin. Hematol. 2018, 25, 29-36. [CrossRef]

71. Quirt, I.C.; Shelley, W.E.; Pater, J.L.; Bodurtha, A.J.; McCulloch, P.B.; McPherson, T.A.; Paterson, A.H.; Prentice, R.; Silver, H.K.; Willan, A.R.; et al. Improved survival in patients with poor-prognosis malignant melanoma treated with adjuvant levamisole: A phase III study by the National Cancer Institute of Canada Clinical Trials Group. J. Clin. Oncol. 1991, 9, 729-735. [CrossRef] [PubMed]

72. Scheinfeld, N.; Rosenberg, J.D.; Weinberg, J.M. Levamisole in dermatology: A review. Am. J. Clin. Derm. 2004, 5, 97-104. [CrossRef] [PubMed]

73. Brunt, T.M.; van den Berg, J.; Pennings, E.; Venhuis, B. Adverse effects of levamisole in cocaine user: A reveiw and risk assessment. Arch. Toxicol. 2017, 91, 2303-2313. [CrossRef] [PubMed]

74. Carmona-Rivera, C.; Purmalek, M.M.; Moore, E.; Waldman, M.; Walter, P.J.; Garrafo, H.M.; Phillips, K.A.; Preston, K.L.; Graf, J.; Kaplan, M.J.; et al. A role for muscarinic receptors in neutrophil extracellular trap formation and levamisole-induced autoimmunity. JCI Insight 2017, 2, e89780. [CrossRef]

75. Prandota, J.; Kobylinska, J.; Lipowska, K. Administration of levamisole to children with kidney disease exacerbation caused by recurrent respiratory infections. Pol. Tyg. Lek. 1989, 44, 673-676. [PubMed]

76. Youssef, D.M.; Abd Al-Atif, A.M.; El-Khateeb, S.S.H.; Elshal, A.S. Evaluation of interleukin-18 in children with steroid-sensitive nephrotic syndrome before and after using levamisole. Saudi J. Kidney Dis. Transpl. 2018, 29, 591-597. [CrossRef]

77. Lombel, R.M.; Gipson, D.S.; Hodson, E.M. Treatment of steroid-sensitive nephrotic syndrome: New guidelines from KDIGO. Pediatr. Nephrol. 2013, 28, 415-426. [CrossRef]

78. Yoo, T.H.; Fornoni, A. Nonimmunologic targets of immunosuppressive agents in podocytes. Kidney Res. Clin. Pract. 2015, 34, 69-75. [CrossRef]

79. Yap, H.K.; Cheung, W.; Murugasu, B.; Sim, S.K.; Seah, C.C.; Jordan, S.C. Th1 and Th2 cytokine mRNA profiles in childhood nephrotic syndrome: Evidence for increased IL-13 mRNA expression in relapse. J. Am. Soc. Nephrol. 1999, 10, 529-537.

80. Kim, J.E.; Park, S.J.; Ha, T.S.; Shin, J.I. Effect of rituximab in MCNS: A role for IL-13 suppression? Nat. Rev. Nephrol. 2013, 9, 551. [CrossRef] 
81. Kim, A.H.; Chung, J.J.; Akilesh, S.; Koziell, A.; Jain, S.; Hodgin, J.B.; Miller, M.J.; Stappenbeck, T.S.; Miner, J.H.; Shaw, A.S. B cell-derived IL-4 acts on podocytes to induce proteinuria and foot process effacement. JCI Insight 2017, 2, e81836. [CrossRef] [PubMed]

82. Lai, K.W.; Wei, C.L.; Tan, L.K.; Tan, P.H.; Chiang, G.S.; Lee, C.G.; Jordan, S.C.; Yap, H.K. Overexpression of interleukin-13 induces minimal-change-like nephropathy in rats. J. Am. Soc. Nephrol. 2007, 18, 1476-1485. [CrossRef] [PubMed]

83. Faul, C.; Donnelly, M.; Merscher-Gomez, S.; Chang, Y.H.; Franz, S.; Delfgaauw, J.; Chang, J.M.; Choi, H.Y.; Campbell, K.N.; Kim, K.; et al. The actin cytoskeleton of kidney podocytes is a direct target of the antiproteinuric effect of cyclosporine A. Nat. Med. 2008, 14, 931-938. [CrossRef] [PubMed]

84. Faul, C.; Asanuma, K.; Yanagida-Asanuma, E.; Kim, K.; Mundel, P. Actin up: Regulation of podocyte structure and function by components of the actin cytoskeleton. Trends Cell Biol. 2007, 17, 428-437. [CrossRef] [PubMed]

85. Funauchi, M.; Ikoma, S.; Enomoto, H.; Horiuchi, A. Decreased Th1-like and increased Th2-like cells in systemic lupus erythematosus. Scand. J. Rheumatol. 1998, 27, 219-224. [CrossRef] [PubMed]

86. Robinson, D.S.; Hamid, Q.; Ying, S.; Tsicopoulos, A.; Barkans, J.; Bentley, A.M.; Corrigan, C.; Durham, S.R.; Kay, A.B. Predominant TH2-like bronchoalveolar T-lymphocyte population in atopic asthma. N. Engl. J. Med. 1992, 326, 298-304. [CrossRef] [PubMed]

87. Kocabas, C.N.; Sekerel, B.E.; Firat, P.A.; Okur, H.; Adahoglu, G. Levamisole: Might it be used in treatment and prevention of atopic diseases? J. Asthma 2004, 41, 547-551. [CrossRef]

88. Heurtier, A.H.; Boitard, C. T-cell regulation in murine and human autoimmune diabetes: The role of TH1 and TH2 cells. Diabetes Metab. 1997, 23, 377-385.

89. Szeto, C.; Gillespie, K.M.; Mathieson, P.W. Levamisole induces interleukin-18 and shifts type 1/type 2 cytokine balance. Immunology 2000, 100, 217-224. [CrossRef]

90. Qian, H.; Jiao, L.; Fan, Z.; Wang, L.; Liu, B.; Miao, G. Analysis of Immunologic Function Changes in Lichen Planus After Clinical Treatment. Med. Sci. Monit. 2018, 24, 8716-8721. [CrossRef]

91. Park, S.J.; Saleem, M.A.; Nam, J.A.; Ha, T.S.; Shin, J.I. Effects of interleukin-13 and montelukast on the expression of zonula occludens-1 in human podocytes. Yonsei Med. J. 2015, 56, 426-432. [CrossRef] [PubMed]

92. Vivarelli, M.; Emma, F. Levamisole for children with nephrotic syndrome: New evidence for the use of an “old" drug. Kidney Int. 2019, 95, 25-28. [CrossRef] [PubMed]

93. Van Wauwe, J.; Janssen, P.A. On the biochemical mode of action of levamisole: An update. Int. J. Immunopharmacol. 1991, 13, 3-9. [CrossRef]

94. Colucci, M.; Carsetti, R.; Cassioli, S.; Serafinelli, J.; Emma, F.; Vivarelli, M. B cell phenotype in pediatric idiopathic nephrotic syndrome. Pediatr. Nephrol. 2019, 34, 177-181. [CrossRef] [PubMed]

95. Wada, T.; Pippin, J.W.; Marshall, C.B.; Griffin, S.V.; Shankland, S.J. Dexamethasone prevents podocyte apoptosis induced by puromycin aminonucleoside: Role of p53 and Bcl-2-related family proteins. J. Am. Soc. Nephrol. 2005, 16, 2615-2625. [CrossRef] [PubMed]

96. Xing, C.Y.; Saleem, M.A.; Coward, R.J.; Ni, L.; Witherden, I.R.; Mathieson, P.W. Direct effects of dexamethasone on human podocytes. Kidney Int. 2006, 70, 1038-1045. [CrossRef] [PubMed]

97. Ransom, R.F.; Lam, N.G.; Hallett, M.A.; Atkinson, S.J.; Smoyer, W.E. Glucocorticoids protect and enhance recovery of cultured murine podocytes via actin filament stabilization. Kidney Int. 2005, 68, 2473-2483. [CrossRef] [PubMed]

98. Tenbrock, K.; Muller-Berghaus, J.; Fuchshuber, A.; Michalk, D.; Querfeld, U. Levamisole treatment in steroid-sensitive and steroid-resistant nephrotic syndrome. Pediatr. Nephrol. 1998, 12, 459-462. [CrossRef]

(C) 2019 by the authors. Licensee MDPI, Basel, Switzerland. This article is an open access article distributed under the terms and conditions of the Creative Commons Attribution (CC BY) license (http://creativecommons.org/licenses/by/4.0/). 